Pacific Journal of Mathematics

THE LÜROTH SEMIGROUP OF PLANE ALGEBRAIC CURVES 


\title{
THE LÜROTH SEMIGROUP OF PLANE ALGEBRAIC CURVES
}

\section{S. Greco and G. Raciti}

\begin{abstract}
A "gap" for a smooth irreducible complete algebraic curve $C$ is a non-negative integer $n$ such that no rational function on $C$ has degree $n$. The non-gaps form the so called "Lüroth semigroup" of $C$.

We give methods to find gaps and non-gaps when $C$ is a plane curve of degree $d$, based on properties of linear series and Hilbert functions. It turns out that for $d \leq 14$ the Lüroth semigroup depends only on $d$; and for larger $d$ we point out where two curves might have different gaps. Bounds are also given for the conductor of the Lüroth semigroup, depending on $d$.
\end{abstract}

Introduction. The Lüroth semigroup (LS) of a smooth irreducible complete algebraic curve $C$ is the additive semigroup $S_{C}$ containing all the degrees of the rational functions of $C$ (equivalently: the degrees of the linear series on $C$ without base points). As such it was introduced by Heinzer and Moh [HM], but the problems related to it are as old as the theory of curves: indeed the knowledge of the degrees of the rational functions on $C$ is a remarkable step towards the knowledge of the geometry of $C$.

A systematic account on $S_{C}$ is available only in a few cases, namely curves with general moduli, hyperelliptic curves, and plane curves up to degree 9 (see 1.3 below).

The main purpose of this paper is to study $S_{C}$ for a plane curve $C$ of degree $d \geq 4$ (the situation being trivial for $d \leq 3$ ). After some preliminaries collected in $\S 1$, we prove, in $\S 2$, that if a curve carries a very ample linear series of degree $m$ and dimension $r$, then all the integers $n$ such that $m-r+1 \leq n \leq m$ belong to its LS (Corollary 2.2). This fact follows from a theorem of Bertini (proved in arbitrary characteristic by Laksov [L]), and implies that, if $C$ is as above, then $S_{C}$ contains all integers $n$ such that $a d-a(a+3) / 2+1 \leq n \leq a d$ for $a \in \mathbb{N}$ and $1 \leq a \leq d-1$ (Corollary 2.6).

In $\S 3$ we show that no integer $n$ with $(a-1) d<n<a d-a^{2}$ $\left(a \in \mathbb{N}\right.$ ) can belong to $S_{C}$ (Theorem 3.1). To prove this we use some results of Davis [D] to study the Hilbert function of a zerodimensional subscheme of $\mathbb{P}^{2}$ contained in $C$; in this way we can 
get control on the indexes of speciality of certain divisors on $C$, and we can conclude, by Riemann-Roch, that every linear series on $C$ of degree $n$ has a base point.

In $\S 4$ we show (Theorem 4.1) that if $3 \leq a \leq d-1$ then $S_{C}$ contains at least an integer $n$ such that $a d-a^{2} \leq n \leq a d-a(a+3) / 2$; in particular we get that $3 d-9$ always belongs to $S_{C}$, thus answering a question raised in [HM]. The proof consists in showing that there is a pencil of curves of degree $a$ with sufficiently many base points on $C$; and this follows from the existence of suitable nets, whose construction is possible by results of Maggioni-Ragusa [MR].

We also show, following a suggestion by P. Ellia, that for any $d \geq$ $2 a-2$ and any $n$ as above there is a curve $C$ of degree $d$ such that $n \in S_{C}$ (at least in characteristic zero, Proposition 4.3).

The above results on plane curves are summarized in $\S 5$ (Theorem 5.1 , where they are used to give bounds on the conductor of $S_{C}$ (Corollary 5.3), and to compute $S_{C}$ when $d \leq 14$ : in this case it turns out that $S_{C}$ depends only on $d$ (Corollary 5.5).

We wish to thank W. Heinzer for bringing our attention to these problems, and M. Boratynski for many helpful discussions. Our thanks go also to the Department of Mathematics of Purdue University and to the Mittag-Leffler Institute for hospitality and support to the first author during some preliminary work on the subject of this paper.

1. Preliminaries. We shall denote by $C$ a smooth irreducible complete curve of genus $g$ over an algebraically closed field $k$ of arbitrary characteristic. We denote by $k(C)$ the field of rational functions on $C$, and we use freely the basic facts on divisor and linear series on $C$ (see $[\mathbf{H}]$ for a reference). We denote by $\mathbb{P}^{r}$ the projective $r$-dimensional space over $k$.

1.1. Definition. A non-negative integer $n$ is said to be a "gap" if every linear series of degree $n$ on $C$ has at least a base point.

The set of non-gaps is denoted by $S_{C}$, and is an additive sub-semigroup of $\mathbb{N}$ (as one shows easily by using the sum of linear series).

Following [HM] we call $S_{C}$ the "Lüroth semigroup" (shortly LS) of $C$; the motivation for this name can be found in [HM].

1.2. REMARK. It is easy to show that the following are equivalent for a given $n \in \mathbb{N}$

(i) $n \in S_{C}$ (i.e. there is a $g_{n}^{r}$ without base points). 
(ii) There exists a $g_{n}^{1}$ without base points.

(iii) There is a rational function $f \in k(C)$ of degree $n$ (i.e. the divisor of zeros (or poles) of $f$ has degree $n$ ).

(iv) There is a finite morphism $C \rightarrow \mathbb{P}^{1}$ of degree $n$.

(v) There is an invertible sheaf on $C$ of degree $n$ and generated by global sections.

1.3. Examples. (i) $S_{C}=\mathbb{N}$ if and only if $C \simeq \mathbb{P}^{1}$.

(ii) If $g=1$ then $n=1$ is the only gap.

(iii) If $g \geq 2$ and $C$ is hyperelliptic then $S_{C}$ is generated by 2 , $g+1, g+2$ (see [HM]).

(iv) $S_{C}$ is known for every plane curve $C$ of degree $d \leq 9$ (see [HM]). We shall see later how to compute $S_{C}$ for $d \leq 14$.

(v) It is known that if $C$ has "general moduli" then $S_{C}=\{n \in$ $\mathbb{N} \mid n \geq(g+1) / 2\}$ (in characteristic zero, see e.g. [HO], introduction).

2. An elementary method to find non-gaps. If we know that $n \in S_{C}$, i.e. that there is a $\sigma=g_{n}^{r}$ without base points, we can try to consider the residual series of $\sigma$ with respect to a general point $P$, to get a $g_{n-1}^{r-1}$. If this linear series happens to be without base points, then we get $n-1 \in S_{C}$. This elementary idea can be carried out in several cases, giving in a unified way a number of (mostly well-known) facts. Our argument is based on the following lemma.

2.1. Lemma. Let $\varphi: C \rightarrow C^{\prime} \subset \mathbb{P}^{r}, r \geq 2$, be a birational morphism, where $C^{\prime}$ is a (possibly singular) curve of degree $n$ not contained in a hyperplane.

Then:

(a) $n, n-1 \in S_{C}$.

(b) If $C^{\prime}$ is non-strange and $r \geq 3$ then $n-2, \ldots, n-r+1 \in S_{C}$. (See $[\mathbf{H}]$, p. 311 for the definition of a strange curve.)

Proof. (a) The hyperplanes (resp. the hyperplanes through a general point $P \in C^{\prime}$ ) determine, by pull-back, a linear series without base points of degree $n$ (resp. $n-1$ ).

(b) If $C^{\prime}$ is non-strange and $i$ is an integer such that $2 \leq i \leq r-1$ then by [L], Lemma 1 there are non-singular distinct points $P_{1}, \ldots, P_{i}$ on $C^{\prime}$ such that the linear space $L$ they span determines on $C$ exactly the divisor $D=P_{1}+\cdots+P_{i}$ (we identify $P_{i}$ with $\varphi^{-1}\left(P_{i}\right)$ ).

Thus the hyperplanes through $P_{1}, \ldots, P_{i}$ cut out on $C$, outside of $D$, a linear series of degree $n-i$ without base points. 
2.2. Corollary. Let $\sigma=g_{n}^{r}$ be a linear series on $C$ without base points, and let $\varphi: C \rightarrow \mathbb{P}^{r}$ be the corresponding morphism. Assume that $r \geq 2$ and that $\varphi: C \rightarrow C^{\prime}:=\varphi(C)$ is birational. Then

(a) $n, n-1 \in S_{C}$.

(b) If either $\operatorname{char}(k)=0$ or $\sigma$ is very ample then $n, n-1, \ldots$, $n-r+1 \in S_{C}$.

Proof. By assumption $C^{\prime}$ is a non-degenerate curve of degree $n$. Then (a) follows immediately from 2.1 (a). To prove (b) we may assume $r \geq 3$ (otherwise apply (a)). Then $C^{\prime}$ is neither a line nor a conic, and hence $C^{\prime}$ is non-strange $([\mathbf{H}]$, Theorem 3.9), and the conclusion follows from (a) and 2.1(b).

2.3. REMARK. In characteristic zero the existence of $P_{1}, \ldots, P_{i}$ as in the proof of 2.1 is a classical theorem of Bertini. Thus in characteristic zero 2.1 and 2.2 are much simpler to state and to prove.

2.4. Corollary. (i) If $n \geq g+1$ then $n \in S_{C}$.

(ii) If $C$ is non-hyperelliptic then $g \in S_{C}$.

Proof. By a theorem of Halphen's ([H], Prop. 6.1) for any $n \geq g+3$ $C$ has a very ample linear series of degree $n$. Then (i) follows from 2.2.

Similarly if $C$ is non-hyperelliptic the canonical series is a very ample linear series of degree $2 g-2$ and dimension $g-1$, and (ii) follows again by 2.2 .

2.5. REMARKs. (i) Corollary 2.4 is well known in characteristic zero (e.g. [ACGH]), but we are not aware of any proof in positive characteristic.

(ii) If $C$ is non-hyperelliptic of genus 3 then $2=g-1 \notin S_{C}$ : this follows easily by considering $C$ as a non-singular plane quartic (see Theorem 3.1 below for details). On the other hand if $g \geq 4$ then $g-1 \in S_{C}([\mathbf{A C G H}]$, p. 372, F). For further information on the gaps "near $g$ " see e.g. [HO].

2.6. Corollary. Assume $C$ is a plane smooth curve of degree $d$, and let $a$ be an integer such that $1 \leq a \leq d-1$. Then every integer $n$ such that ad $-a(a+3) / 2+1 \leq n \leq a d$ belongs to $S_{C}$.

Proof. The linear series cut out on $C$ by the curves of degree $a$ has dimension $a(a+3) / 2$ and degree $a d$. Moreover it is easily seen to be every ample. The conclusion follows then by $2.2(\mathrm{~b})$. 
3. Finding gaps on a plane curve. In $\S 2$ we have shown that certain sequences of integers always belong to $S_{C}$, where $C$ is a plane curve of degree $d$.

In this section we find something opposite: namely we produce sequences of integers, none of which belong to $S_{C}$. The main result is Theorem 3.1, whose proof is based on the study of the Hilbert function of certain zero-dimensional subschemes of $\mathbb{P}^{2}$.

3.1. TheOREM. If $C \subset \mathbb{P}^{2}$ is a non-singular curve of degree $d \geq 4$ and $(a-1) d+1 \leq n \leq a d-\left(a^{2}+1\right)$, with $a \in \mathbb{N}$ and $a \geq 1$, there is no $g_{n}^{r}$ on $C$ without base points (i.e. $n \notin S_{C}$ ).

3.2. REMARK. It is only a matter of computation to show that $n$ as above exists if and only if $d \geq a^{2}+2$ and that $a d-\left(a^{2}+1\right) \leq g-1$ if $a \geq 1$ and $d \geq a^{2}+2$. So 3.1 agrees with Corollary 2.4(ii).

Before giving the proof of Theorem 3.1 we recall some basic facts about the Hilbert function of a zero-dimensional closed subscheme of $\mathbb{P}^{2}$.

3.3. Some basic facts about Hilbert functions. If $X \subset \mathbb{P}^{2}$ is a zerodimensional closed subscheme of degree $\delta(X)=n$, we denote by $H(X, i)$ the Hilbert function of $X$ and by $\Delta H(X, i)=H(X, i)-$ $H(X, i-1)$ its first difference, for every $i>0, H(X, 0)=\Delta H(X, 0)$ $=1$.

It is well known that, if $\alpha$ is the least degree of a curve through $X$, $\Delta H(X,-)$ is characterized by the following properties (see [D]):

$$
\begin{gathered}
\Delta H(X, i)=i+1 \quad \text { for } 0 \leq i \leq \alpha-1, \\
\Delta H(X, i) \geq \Delta H(X, i+1) \quad \text { for } i \geq \alpha, \\
\Delta H(X, i) \text { is eventually zero, } \\
H(X, s)=\sum_{i=0}^{s} \Delta H(X, i) ; \quad \text { in particular } \\
\delta(X)=\sum_{i=0}^{\infty} \Delta H(X, i)=H(X, s) \quad \text { for } s \gg 0 .
\end{gathered}
$$

Moreover it will be important to know

$$
\text { If } X^{\prime} \subset X \text { then } \Delta H\left(X^{\prime}, i\right) \leq \Delta H(X, i) \text { for every } i \geq 0
$$

(see [D] or, for more details, [R]). 
In the following we will set $\Delta H(X, i)=c_{i}$ and $t=\max \{i \in \mathbb{N}\}$ $\left.c_{i} \neq 0\right\}$.

Let $s$ be an integer such that $\alpha \leq s \leq t, \Gamma_{s}$ any curve of $\mathbb{P}^{2}$ of degree $c_{s}$ and $\Sigma_{s}=\Sigma_{s}(X)$ the linear system of curves of degree $s$ through $X$.

3.4. LEMMA. With the previous notation we have:

$$
\delta\left(X \cap \Gamma_{s}\right) \leq c_{s} \cdot s-\frac{c_{s}\left(c_{s}-3\right)}{2}+\sum_{i=s+1}^{t} c_{i} .
$$

(2) If there exists $s$ such that $c_{s-1}=c_{s}$ then $\Sigma_{s}$ has a fixed component $\Gamma$ of degree $c_{s}$ and:

$$
\delta(X \cap \Gamma)=c_{s} \cdot s-\frac{c_{s}\left(c_{s}-3\right)}{2}+\sum_{i=s+1}^{t} c_{i} .
$$

Proof. (1) follows from [D] (2.2)(a) if we observe that by (3.3.2), as $s \geq \alpha$, it is $c_{s} \leq s$.

(2) follows from [D] (2.2)(b) and (2.3).

3.5. LemMA. Let $X$ be a zero dimensional subscheme of $\mathbb{P}^{2}$ and suppose that $0<c_{j-1}=c_{j}=h<\alpha$ for some integer $j$. Then there exists a subscheme $Y \subset X$ such that:

$$
\begin{gathered}
\delta(Y)=\delta(X)-1 \\
H(Y, i)=H(X, i)-1 \quad \text { for every } i \geq j-2 .
\end{gathered}
$$

Proof. By (3.3.4) it is equivalent to show that there exists $Y \subset X$, with $\delta(Y)=\delta(X)-1$, such that $\Delta H(Y, i)=c_{i}-1$ for some $i \leq j-2$. If we denote by $s$ the least integer for which $c_{s}=h$, by (2) of Lemma 3.4 , the curves of $\Sigma_{s}$ contain a fixed component $\Gamma$ of degree $h$ and it is

$$
\delta(X \cap \Gamma)=c_{s} \cdot s-\frac{c_{s}\left(c_{s}-3\right)}{2}+\sum_{i=s+1}^{t} c_{i}:=m .
$$

As $c_{S}<\alpha$ we get $\delta(X \cap \Gamma)<\delta(X)$, and hence there is a scheme $Y$ such that $X \cap \Gamma \subset Y \subset X$ and $\delta(Y)=\delta(X)-1$. For such a $Y$ we have $\Delta H(Y, i) \leq c_{i}$ for every $i$ and $\Delta H(Y, i)=c_{i}-1$ for exactly one $i$ respectively by (3.3.5) and (3.3.4). By assumption $c_{s}=c_{s+1}=\cdots=c_{s+k}, k \geq 1$; it can be neither $\Delta H(Y, i)=c_{i}-1$ if $s \leq i \leq s+k-1$ by (3.3.2) nor $\Delta H(Y, i)=c_{i}-1$ if $i \geq s+k$ for 
otherwise Lemma 3.4(1) would imply $\delta(\Gamma \cap X)=\delta(\Gamma \cap Y) \leq m-1$, a contradiction. Then $\Delta H(Y, i)=c_{i}-1$ for some $i<s$.

3.6. Lemma. Let $D$ be an effective divisor on $C$, and let $X \subset \mathbb{P}^{2}$ be the corresponding closed subscheme. Then the following are equivalent:

(1) There exists a closed subscheme $Y \subset X$ such that $H(X, d-3)=$ $H(Y, d-3)+1$ and $\delta(Y)=\delta(X)-1$.

(2) The linear series $|D|$ has a base point.

Proof. (1) $\Rightarrow(2)$. Let $E$ be the divisor of $C$ corresponding to $Y$. Then $E$ is effective and $E=D-P$ for a suitable $P \in C$. Moreover, since the canonical series of $C$ is cut out by the curves of degree $d-3$, we have

$i(D)=\left(\begin{array}{c}d-1 \\ 2\end{array}\right)-H(X, d-3)<\left(\begin{array}{c}d-1 \\ 2\end{array}\right)-H(Y, d-3)=i(D-P)$.

Then, by Riemann-Roch, $P$ is a base point of $|D|$.

$(2) \Rightarrow(1)$. Reverse the above argument.

Proof of Theorem 3.1. Clearly we may assume $d \geq a^{2}+2$, for otherwise $n$ does not exist.

Let $D$ be an effective divisor of degree $n$; we have to show that the linear series $|D|$ has a base point i.e., by Lemma 3.6, that if $X$ is the closed subscheme of $\mathbb{P}^{2}$ corresponding to $D$, there is a closed subscheme $Y \subset X$ of degree $n-1$, such that $H(X, d-3)=$ $H(Y, d-3)+1$. We argue by contradiction.

So we assume:

(a) $H(X, d-3)=H(Y, d-3)$ for every subscheme $Y \subset X$ of degree $n-1$.

If $Y$ is any subscheme of $X$ of degree $n-1$ we have then $n-1 \geq$ $H(Y, d-3)=H(X, d-3)$ which implies:

(b) $\Delta H(X, d-2)>0$,

for otherwise, by (3.3.2) and (3.3.4), $H(X, d-2)=H(X, d-3)=$ $\delta(X) \leq n-1$.

If $a=1$, we have $n \leq d-2$ and then $\Delta H(X, d-2)=0$ by (3.3.1), (3.3.2) and (3.3.4), contrary to (b).

So we may assume $a \geq 2$.

Now if $\alpha$ is the least degree of a curve containing $X$ we have $a \leq \alpha$ by Bezout, whence $\Delta H(X, a-1)=a$. It follows:

(c) if $s$ is any integer such that $a-1<s<d-2$ and $\Delta H(X, s)<a$, then

$$
\Delta H(X, s)>\Delta H(X, s+1)>\cdots>\Delta H(X, d-2)>0 .
$$


Indeed if $\Delta H(X, i)=\Delta H(X, i+1)$ for some $s \leq i \leq d-3$ by Lemma 3.5 there exists $Y \subset X$ such that $\delta(Y)=\delta(X)-1$ and $H(Y, i)=H(X, i)-1$ for every $i \geq s-1$, in particular $H(Y, d-3)=$ $H(X, d-3)-1$ against (a).

We shall get a contradiction by showing that (c) implies:

(d) $\Delta H(X, i) \geq \Delta H(Z, i)$ for all $i$ 's, where $Z$ is a complete intersection of type $(a, d-a)$.

Indeed if (d) holds, by (3.3.4) and (3.3.5) we have $n \geq a(d-a)$ which is absurd.

To show that (c) implies (d) recall first that, since $d-a>a$ (as follows easily from $d \geq a^{2}+2$ ), from the free resolution of the homogeneous saturated ideal of $Z$ we have:

$$
\Delta H(Z, i)= \begin{cases}i+1 & \text { for } 0 \leq i \leq a-1 \\ a & \text { for } a \leq i \leq d-a-1 \\ d-i-1 & \text { for } d-a \leq i \leq d-2 \\ 0 & \text { for } i \geq d-1\end{cases}
$$

As $a \leq \alpha$, (d) holds for $0 \leq i \leq a-1$ by (3.3.1) ((c) is not used here). Next observe that (c) implies

$$
\Delta H(X, d-2-j) \geq \min \{a, j+1\}
$$

for all $j$ such that $d-2-j>a-1$ and in particular for $j=a-1$ since $d>2 a$. This implies (d) for $d-a-1 \leq i<\infty$. Finally for $a \leq i \leq d-a-2$ the inequality (d) follows easily from (3.3.2). Thus (c) implies (d) and the proof is complete.

4. Finding non gaps on a plane curve. The sequences of gaps and non-gaps for a plane curve $C$ of degree $d$ found in 3.1 and 2.6 leave out the integers $n$ such that $a d-a^{2} \leq n \leq a d-a(a+3) / 2,3 \leq a \leq$ $d-1$.

In this section we show that, for given $a$, at least an integer as above belongs to $S_{C}$ (Theorem 4.1), and that if $d \geq 2 a-2$ then for any $n$ as above there is a curve $C$ of degree $d$ having $n$ as a non-gap (characteristic zero, see 4.3).

4.1. THEOREM. If $C \subset \mathbb{P}^{2}$ is a non-singular curve of degree $d$ and $a$ is an integer such that $d>a \geq 3$ there is at least an $n \in S_{C}$ such that $a d-a^{2} \leq n \leq a d-a(a+3) / 2$. 
Proof. Since $d>a$, we can choose on $C$ a set $X$ of $\left(\begin{array}{c}a+2 \\ 2\end{array}\right)-3:=k$ distinct points such that:

(1) no $\left(\begin{array}{c}a^{\prime}+2 \\ 2\end{array}\right)$ among the $k$ points, $1 \leq a^{\prime} \leq a-1$, lie on a curve of degree $a^{\prime}$.

(2) $\operatorname{dim} \Sigma_{a}=\left(\begin{array}{c}a+2 \\ 2\end{array}\right)-1-k=2$.

This follows from the proof of Lemma 3.3. in [MR]. Moreover, (cfr. [GM], Theorem 3.4), as $a \geq 3$, every curve in $\Sigma_{a}(X)$ is irreducible hence every pencil contained in $\Sigma_{a}$ has exactly $a^{2}$ base points.

Let $D$ be the divisor on $C$ corresponding to $X$ and $\sigma$ the complete linear series cut out on $C$ by $\Sigma_{a}(X)$ outside of $D$. Note that $\sigma$ has dimension two.

If $\sigma$ is without base points it cannot be very ample otherwise the projective image of $C$ by $\sigma$ would be a non-singular plane curve of degree $a d-k$ and then, by the genus formula, we would have $a d-k=d$, i.e $d=(a+4) / 2$, which is absurd.

Hence there exists a divisor $P+Q$ on $C$ such that

$$
\operatorname{dim}|\sigma-(P+Q)|=1 \text {. }
$$

So there are two different curves $C_{1}$ and $C_{2}$ in $\Sigma_{a}(X)$ such that $C_{i} \cdot C \geq D+P+Q, i=1,2$.

If $\sigma$ has base points the same follows easily. Moreover, as $C$ is non-singular, we can see that $i\left(A, C_{1} \cap C_{2}\right) \geq \min i\left(A, C_{i} \cap C\right), i=$ 1,2 and $A$ any point on $C$. (This follows from [F], Ch. $3 \S 3$; see [C], Lemma 5.2 for details.) Then if $E$ is the largest divisor such that $0 \leq E \leq C_{i} \cdot C$ for $i=1,2$, and $h=\operatorname{deg} E$, we have:

$$
\frac{a(a+3)}{2}=\operatorname{deg} D+2 \leq h \leq a^{2} .
$$

Hence the divisors $D_{i}=C_{i} \cdot C-E$ are linearly equivalent and have disjoint support. Hence they belong to a $g_{a d-h}^{1}$ without base point; and since $a d-a^{2} \leq a d-h \leq a d-a(a+3) / 2$ our proof is complete.

4.2. Corollary. If $d \geq 4$ then $3 d-9 \in S_{C}$.

Proof. Apply Theorem 4.1 with $a=3$.

4.3. Proposition. If $\operatorname{char}(k)=0$ and $a d-a^{2} \leq n \leq a d-$ $a(a+3) / 2, a \geq 3$, then for every $d \geq 2 a-2$ there exists a curve $C$ of degree $d$ such that $n \in S_{C}$.

Proof. Let $\Gamma$ and $\Gamma^{\prime}$ be two non-singular curves of degree $a$ which intersect in $a^{2}$ distinct points $P_{1}, \ldots, P_{a^{2}}$. Then, by considering the 
Hilbert function of a complete intersection of type $(a, a)$ we see that $2 a-2$ is the least degree of a curve to which they impose independent conditions. Hence, if we fix $d \geq 2 a-2$ and $k$ points $P_{1}, \ldots, P_{k}$ among $P_{1}, \ldots, P_{a^{2}}, a(a+3) / 2 \leq k=a d-n \leq a^{2}$, there always exists a curve $C$ of degree $d$ which contains these $k$ points and no one of the other $a^{2}-k$. Now we prove that such a $C$ can be found non-singular. So we consider the linear system $\Sigma$ of curves of degree $d$ through $P_{1}, \ldots, P_{k}$. Since $a \geq 3$ we have $d \geq 2 a-2>a$ and then all the curves having $\Gamma$ or $\Gamma^{\prime}$ as a component belong to $\Sigma$. Then the base locus of $\Sigma$ is contained in the set of points $P_{1}, \ldots, P_{a^{2}}$ and so, by the above argument, it is exactly $\left\{P_{1}, \ldots, P_{k}\right\}$. Then, by Bertini's theorem, the generic curve of $\Sigma$ is non-singular off of $P_{1}, \ldots, P_{k}$. The same argument shows that $\Sigma$ contains some curve non-singular in $P_{1}, \ldots, P_{k}$ and then the generic curve in $\Sigma$ is non-singular.

So the pencil generated by $\Gamma$ and $\Gamma^{\prime}$ cuts out on $C$, outside of $P_{1}, \ldots, P_{k}$, a $g_{a d-k}^{1}$ without base points.

4.4. REMARK. If $d \gg a \geq 3$ we can find a curve $C$ of degree $d$ such that $n \in S_{C}$ for every $n \in\left[a d-a^{2}\right.$, ad $\left.-a(a+3) / 2\right]$.

We can argue as in the proof of the above proposition if we make the following construction. First we fix $t+1$ curves $\Gamma, \Gamma_{1}, \ldots, \Gamma_{t}$ of degree $a$, where $t$ is the number of integers in the interval we are considering, and we choose them in such a way that $\Gamma \cap \Gamma_{i}, i=$ $1 \ldots, t$, are disjoint sets $X_{i}$ of $a^{2}$ distinct points. Then we fix subsets $Y_{i}$ of $X_{i}$ in order to obtain all the integers in $\left[a d-a^{2}, a d-a(a+3) / 2\right]$ and finally we consider the curve of degree $d$ through $Y_{1} \cup Y_{2} \cup \cdots \cup Y_{n}$.

This construction works, if $d$ is very large with respect to $a$.

5. The Lüroth semigroup of a plane curve and its conductor. In this section we summarize all the results we know on the Lüroth semigroup of a plane curve $C$ of degree $d$, and we apply them to give a lower and upper bound for the conductor of $S_{C}$ in terms of $d$, and to compute $S_{C}$ for $d \leq 14$.

If $d=1,2,3$ then $C$ is rational or elliptic, and the situation is clear, see 1.3. For $d \geq 4$ we can summarize $2.6,3.1$ and 4.1 in the following theorem.

5.1. Theorem. Let $C$ be a smooth plane curve of degree $d \geq 4$ and let $a$ be an integer, $1 \leq a \leq d-1$. Then:

(a) If $(a-1) d+1 \leq n \leq a d-a^{2}-1$ then $n \notin S_{C}$. 
(b) If $a \geq 3$ there is at least an integer $n \in S_{C}$, such that ad $-a^{2} \leq$ $n \leq a d-a(a+3) / 2$. In particular $3 d-9 \in S_{C}$.

(c) If ad $-a(a+3) / 2+1 \leq n \leq$ ad then $n \in S_{C}$.

5.2. REMARKS. (i) As remarked before statement (a) of 5.1 is void iff $d \leq a^{2}+1$.

(ii) Statement (b) is really meaningful if $d \geq a(a+3) / 2+1$. Indeed if $d \leq a(a+3) / 2$ by a direct calculation we have

$$
\begin{gathered}
(a-1) d-(a-1)(a-2) / 2+1 \leq a d-a^{2}, \\
a d-a(a+3) / 2 \leq(a-1) d,
\end{gathered}
$$

and then (b) follows from (c), applied with $a$ replaced by $a-1$.

Recall that if $S \subset \mathbb{N}$ is an additive semigroup, the conductor of $S$ is the least integer $c$ such that every $n \geq c$ belongs to $S$.

If $C$ is a curve we denote by $\gamma_{C}$ the conductor of $S_{C}$.

\subsection{Corollary. Let $d \geq 4$ and put}

$$
\begin{aligned}
& b=\min \{a \in \mathbb{N} \mid a(a+3) / 2 \geq d-1\}, \\
& c=\max \left\{a \in \mathbb{N} \mid a^{2}+2 \leq d-1\right\} .
\end{aligned}
$$

Then:

$$
c d-c^{2} \leq \gamma_{C} \leq b d-b(b+3) / 2+1 \text {. }
$$

Proof. By 5.1.(a) we have $c d-c^{2}-1 \notin S_{C}$, and this gives the first inequality.

Moreover $b(b+3) / 2 \geq d-1$, and hence by $5.1\left(\right.$ c) $S_{C}$ contains at least $d-1$ consecutive integers, namely:

$$
\{n \in \mathbb{N} \mid b d-b(b+3) / 2+1 \leq n \leq b d\} .
$$

And since $d-1 \in S_{C}$, we easily get the second inequality.

5.4. REMARKS. (i) The upper bound for the conductor given in 5.2 was first proved by M. Boratynski (unpublished), by a different method.

(ii) From 5.2, by a direct calculation, one gets

$$
\gamma_{C} \leq \frac{1}{2}(d \sqrt{8 d+1}-5 d+6) \text {. }
$$


This shows that for $d \gg 0, \gamma_{C}$ is bounded above by an integer of order $\sqrt{2} d^{3 / 2}$, whence $\gamma_{C} \ll g-1$. This should be computed with 1.3 (ii), 1.3 (v) and 2.5 (ii).

Now we turn our attention to the LS of $C$ for small $d$ 's. By a straightforward calculation we get, from 5.1 and 5.3:

5.5. Corollary. If $d \leq 14$ then $S_{C}$ depends only on $d$, and is given by the table below.

\begin{tabular}{rl}
\hline$d$ & $\mathbf{N} \backslash S_{C}$ \\
\hline 1,2 & $\varnothing$ \\
3 & 1 \\
4 & 1,2 \\
5 & $1,2,3$ \\
6 & $1, \ldots, 4 ; 7$ \\
7 & $1, \ldots, 5 ; 8,9$ \\
8 & $1, \ldots, 6 ; 9, \ldots, 11$ \\
9 & $1, \ldots, 7 ; 10, \ldots, 13$ \\
10 & $1, \ldots, 8 ; 11, \ldots, 15$ \\
11 & $1, \ldots, 9 ; 12, \ldots, 17 ; 23$ \\
12 & $1, \ldots, 10 ; 13, \ldots, 19 ; 25,26$ \\
13 & $1, \ldots, 11 ; 14, \ldots, 21 ; 27, \ldots, 29$ \\
14 & $1, \ldots, 12 ; 15, \ldots, 23 ; 29, \ldots, 32$ \\
\hline
\end{tabular}

5.6. REMARKs. (i) Corollary 5.5 was proved in [HM] for $d \leq 9$, by different methods.

(ii) It is not clear to us if Corollary 5.5 holds for $d>14$. The first unknown situation occurs for $d=15$, where the only dubious integer is $46=a d-a(a+3) / 2$ with $a=4$ (see 5.1$)$. 
Note however that $46 \in S_{C}$ for some curve $C$ of degree 15 (see Proposition 4.3).

(iii) If for $d \gg 0$ the LS of a curve of degree $d$ is not independent on $d$, it is natural to ask for the structure of $S_{C}$ where $C$ is a general curve of degree $d$. This makes sense because almost all the curves in an irreducible family have the same LS, see [G].

(iv) It is reasonable to believe, as suggested to us by A. Hirschowitz, that for a general curve $C$ of degree $d$ the integers of the form $a d-$ $a(a+3) / 2$ belong to $S_{C}$. Some intuitive evidence for this to be ture is given by our proof of 4.1 .

Added in Proof. We have recently found out that the interesting preprint (to appear in J. Algebra) Free linear systems on integral Gorenstein curves by Marc Coppens contains a proof of our Theorem 3.1 for arbitrary integral plane curves, in the context of Hartshorne's theory of generalized divisors (R. Hartshorne, Generalized divisors on Gorenstein curves and a theorem of Noether, J. Math. Kyoto Univ., 26 (1986), 375-386). Coppens' result clearly implies ours, but his approach to the problem is completely different. It might be interesting to know what happens if one considers Cartier divisors instead.

\section{REFERENCES}

[ACGH] E. Arbarello, M. Cornalba, P. Griffiths and J. Harris, Geometry of Algebraic Curves I, Grund. der Math. Wiss., 267, Springer, New York-Berlin 1985.

[C] L. Caire, Plane curves as projection of non singular space curves, Manuscripta Math., 67 (1990), 433-450.

[D] E. Davis, 0-dimensional subscheme of $\mathbb{P}^{2}$ : New application of Castelnuovo's function. Ann. Univ. Ferrara. Sez VII-Sc. Mat., 32 (1986), 93-107.

[F] W. Fulton, Algebraic Curves, Benjamin, New York, 1969.

[GM] A. Geramita and P. Maroscia, The ideal of forms vanishing at a finite set of points in $\mathbb{P}^{n}$, J. Algebra, 90 (1984), 528-555.

[G] S. Greco, Orders of rational function on curves in a flat family, (preprint).

[H] R. Hartshorne, Algebraic Geometry, GTM 52, Springer-Verlag, New YorkBerlin, 1977.

[Ho] R. Horiuchi, Gap orders of meromorphic functions on Riemann surfaces, J. Reine Angew. Math., 336 (1982), 213-220.

[HM] W. Heinzer and T. T. Moh, On the Lüroth semigroup and Weirstrass canonical divisors, J. Algebra, 77 (1982), 62-73.

[L] D. Laksov, Indecomposability of restricted tangent bundles, Asterisque, 8788 (1980), 207-219.

[MR] R. Maggioni and A. Ragusa, Non singular curves passing through points of $\mathbb{P}^{2}$ in generic position I, J. Algebra, 92 (1985), 176-193. 
[R] G. Raciti, Sulla funzione di Hilbert di un sottoschema zero-dimensionale di $\mathbb{P}^{3}$, Ann. Univ. Ferrara, Ser. VII-Sc. Mat., 35 (1989), 99-112.

Received February 28, 1990.

POLITECNICO DI TORINO

Corso Duca degli Abruzzi, 24

10129 TORINO (ItALY)

AND

UNIVERSITÀ di CATANIA

VIALE A. DORIA, 6

95125 CATANia (ITALY) 


\section{PACIFIC JOURNAL OF MATHEMATICS EDITORS}

\author{
V. S. VARADARAJAN \\ (Managing Editor) \\ University of California \\ Los Angeles, CA 90024-1555-05 \\ Herbert Clemens \\ University of Utah \\ Salt Lake City, UT 84112 \\ THOMAS ENRIGHT \\ University of California, San Diego \\ La Jolla, CA 92093
}

Nicholas ERCOLANI

University of Arizona

Tucson, AZ 85721

R. FINN

Stanford University

Stanford, CA 94305

VAUGHAN F. R. JONES

University of California

Berkeley, CA 94720

STEVEN KeRCKHOFF

Stanford University

Stanford, CA 94305
C. C. MOORE

University of California

Berkeley, CA 94720

Martin ScharlemanN

University of California

Santa Barbara, CA 93106

HAROLd STARK

University of California, San Diego

La Jolla, CA 92093

\section{ASSOCIATE EDITORS}
R. ARENS
E. F. BECKENBACH
B. H. NeUMANN
F. WolF
K. Yoshida (1906-1982)
(1904-1989)

SUPPORTING INSTITUTIONS

UNIVERSITY OF ARIZONA

UNIVERSITY OF BRITISH COLUMBIA

CALIFORNIA INSTITUTE OF TECHNOLOGY

UNIVERSITY OF CALIFORNIA

MONTANA STATE UNIVERSITY

UNIVERSITY OF NEVADA, RENO

NEW MEXICO STATE UNIVERSITY

OREGON STATE UNIVERSITY
UNIVERSITY OF OREGON

UNIVERSITY OF SOUTHERN CALIFORNIA

STANFORD UNIVERSITY

UNIVERSITY OF HAWAII

UNIVERSITY OF TOKYO

UNIVERSITY OF UTAH

WASHINGTON STATE UNIVERSITY

UNIVERSITY OF WASHINGTON 


\section{Pacific Journal of Mathematics}

\section{Vol. 151, No. $1 \quad$ November, 1991}

Fernanda Maria Botelho, Rotational entropy for annulus endomorphisms . . . 1

David F. Cowan, Some infinite chains in the lattice of varieties of inverse

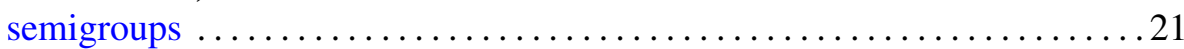

S. Greco and G. Raciti, The Lüroth semigroup of plane algebraic curves . . . 44

Kang-Tae Kim, Automorphism groups of certain domains in $\mathbf{C}^{n}$ with a

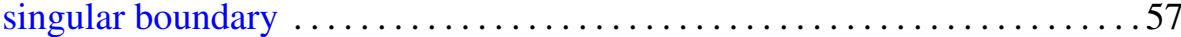

R. James Milgram, Surgery with finite fundamental group. I: The

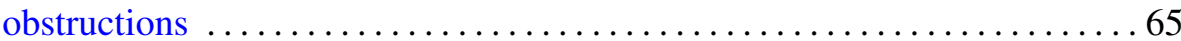

R. James Milgram, Surgery with finite fundamental group. II: The oozing

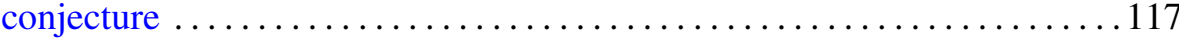

Shahriar Shahriari, On central type factor groups $\ldots \ldots \ldots \ldots \ldots \ldots \ldots 151$

Lynne Walling, Hecke eigenforms and representation numbers of quadratic forms ............................................... 179 